\title{
Periodic Cycles in the Solow Model with a Delay Effect
}

\author{
Anatolij Kulikov ${ }^{a}$, Dmitrij Kulikov ${ }^{a}$ and Michael Radin ${ }^{b}$ \\ ${ }^{a}$ Demidov Yaroslavl State University \\ Sovetskaya st., 14, 150003 Yaroslavl, Russia \\ ${ }^{b}$ Rochester Institute of Technology \\ Rochester,14623 New York, USA \\ E-mail(corresp.): kulikov_d_a@mail.ru
}

Received September 10, 2018; revised March 3, 2019; accepted March 3, 2019

\begin{abstract}
The three natural modifications of the known mathematical macroeconomics model of macroeconomics are studied in which a delay factor is presumed. This led to the replacement of the ordinary differential equation, which cannot exhibit periodic cycles on the equations with a deviating argument (functional-differential equations). It was possible to show the existence of periodic solutions that can and are intended to describe the periodic cycles in the market economy in two of the three variants of such changes in the classical form of the model.

The mathematical portion is based on the application of the modern theory of dynamical systems with an infinite-dimensional space of initial conditions. This will allow us to apply the Andronov-Hopf Theorem for equations with a deviating argument in such a form that the parameters of the cycles are located. We will also apply the well-known Krylov-Bogolyubov algorithm that is extended to infinitedimensional dynamical systems that is used and reduces the problem to the analysis of the finite-dimensional system of ordinary differential equations-the normal PoincareDulac form.
\end{abstract}

Keywords: Solow model, functional-differential equations, stability, bifurcations, normal form, asymptotic formulas.

AMS Subject Classification: 35K18; 37N40.

\section{Introduction}

In macroeconomics, there are a large number of mathematical models that are traditionally considered classical and basic. Traditionally among them, a mathematical model proposed by the Nobel Prize winner in economics R. M. Solow 
[21] (see also [22]). This model is usually called the Solow model, less often Solow-Swan.

This model describes the dynamics of the variable $p=p(\tau)$, where $\tau$ is time. This variable is frequently called the variable capital - labor in economic literature $[2,3,4,5,23,24,25]$. It is usually defined as the ratio $p=K / L$, where $K=K(\tau)$ denotes the physical capital, and $L=L(\tau)$ denotes the number of people employed in the economy. For example, recall in [25], the dynamics of the variable $p=p(\tau)$ can be described by the following equation

$$
p^{\prime}=-\alpha p+\beta p^{k}
$$

where $\alpha, \beta, k$ are positive constants.

Note that a different version of such an equation is possible with a more general form of the right-hand side (see $[23,25]$ )

$$
p^{\prime}=-\alpha p+f(p),
$$

where $f(p)$ is a monotonically increasing convex function. In our case, $f(p)=$ $p^{k}$ and if satisfies the necessary requirements if $k \in(0,1)$.

After replacements (normalizations)

$$
\begin{aligned}
& \tau=\gamma_{0} t, \quad p=\gamma_{1} x, \quad \gamma_{0} \alpha=1, \quad \beta \gamma_{0} \gamma_{1}^{k-1}=1, \\
& \gamma_{0}=1 / \alpha, \quad \gamma_{1}=(\alpha / \beta)^{1 /(k-1)}, \quad \beta, \gamma_{0}, \gamma_{1}>0 .
\end{aligned}
$$

Equation (1.1) acquires the following form:

$$
\dot{x}=-x+x^{k}, \quad x=x(t) .
$$

The first terms on the right-hand side of equations (1.1)-(1.2) are responsible for the depreciation of the capital/labour stock. The second terms are proportional to the gross investment. Next we will consider equation (1.1) for the normalized function $x(t)>0$, via equation (1.2).

Observe that equation (1.2) has a unique positive equilibrium state $x(t)=1$ if $k \neq 1$. Then for $k \in(0,1)$, this equilibrium state is globally asymptotically stable (all solutions of the equation (1.2) with positive initial conditions converge to a given equilibrium position).

In this particular model, it is usually assumed that $k \in(0,1)$ is a stable economic equilibrium $x=1$. Notice that when $k \in(1, \infty)$, the positive equilibrium state $x=1$ is unstable. In the view of most economists, this option of choosing $k$ is less attractive $[23,25]$. For $k=1$, we obtain a completely inconsistent version of equation (1.2) $(\dot{x}=0)$. We also assume that for all $k$, equation (1.1) cannot have stationary solutions with the exception of the equilibrium states. Hence this mathematical model does not quite adequately describe the real economic processes, for which, of course, periods of ups and downs are the characteristic. As a result, equation (1.2) needs modifications. The most natural method is based on the consideration of the delay factor characteristic for economic processes in a market economy (see $[2,3,5,15,16,18,19,23,24])$.

In this paper, we will show that the assumption of the delay factor in the Solow model leads to a meaningful change in the dynamics of the solutions and 
makes it possible to isolate the range of the parameters of the problem under which the periodic cycles exist. From a logical point of view, there are three options for including the lag factor.

The first option is the introduction of delay in the term responsible for the disposal of funds. This option leads to the necessity of analyzing the following equation:

$$
\dot{x}=-y+x^{k}, \quad k \in(0,1),
$$

where $y=y(t)=x(t-h), h>0$. This option is quite sustainable from an economic point of view as it is quite natural to assume that the rate of retirement of fixed assets (depreciation) depends on their value at the previous point in time and on their value during the previous time.

The second option is the introduction of lag involves in the analysis of the following equation:

$$
\dot{x}=-x+y^{k},
$$

where $y=x(t-h)$. The delay is introduced into the second term that is in charge of the investment.

Finally, it is possible that the delay is introduced into both terms, which produces the following equation:

$$
\dot{x}=-y+y^{k},
$$

where as previously $y=x(t-h)$. Naturally, we see that $h>0$.

If we supplement equations (1.3)-(1.5) with the initial condition

$$
x(t)=\varphi(t),
$$

where the given function $x(t) \in C[-h, 0]$ to the space of continuous functions defined on $[-h, 0]$, then we obtain three Cauchy problems: $(1.3),(1.6) ;(1.4)$, $(1.6) ;(1.5),(1.6)$. Each of them generates a local semiflow or in another words a dynamical system in the phase space of solutions.

Observe that equation (1.5) has an equilibrium state $x(t)=1$. In the next section, we will address the stability question. Equation (1.5) can be rewritten in the following form:

$$
\dot{u}=-v+k v+\frac{k(k-1)}{2} v^{2}+\frac{k(k-1)(k-2)}{6} v^{3}+o\left(v^{3}\right),
$$

after replacement which is acquired from equation (1.5) we get

$$
x=1+u, \quad y=1+v, \quad u=u(t), \quad v=v(t)=u(t-h),
$$

and applying the Taylor's formula. Analogous substitutions reduce equation (1.3) to the following form:

$$
\dot{u}=-v+k u+\frac{k(k-1)}{2} u^{2}+\frac{k(k-1)(k-2)}{6} u^{3}+o\left(u^{3}\right),
$$

and equation (1.4) can be replaced by the following substitution:

$$
\dot{u}=-u+k v+\frac{k(k-1)}{2} v^{2}+\frac{k(k-1)(k-2)}{6} v^{3}+o\left(v^{3}\right) .
$$


Notice that all three equations have a zero equilibrium state corresponding to the equilibrium states $x=1$ of the original equations (1.3)-(1.5). Now we will consider the equations (1.7)-(1.9) for which the structure of the neighborhood of the zero equilibrium state will be studied. In the next section, we will review the stability of the zero equilibrium solution for all three previous equations with a deviating argument.

\section{Analysis of linearized equations}

First of all, we will address the stability question of the zero equilibrium state of equation (1.7). To address this question, the analysis of the linearized equation (1.7) will be necessary. In this case we acquire the following equation:

$$
\dot{u}=-v+k v .
$$

In fact, it is well known [7] that stability of solutions of the linear differential equation (2.1) can be reduced to an analysis of the characteristic equation, which in this case exhibits the following form:

$$
\lambda=(k-1) \exp (-\lambda h)
$$

Observe when $h=0$ then we have the root $\lambda=k-1<0$. To find the positive $\min h=H$ for which roots with $\operatorname{Re} \lambda=0$ emerge in Equation (2.2). The case when $\lambda=0$ is not possible for any $h$. Indeed, by substituting $\lambda=0$ in the characteristic equation leads to the equality $k-1=0, k \neq 1(k \in(0,1))$.

Consequently, the critical case in the stability problem is possible. In fact, as follows: for the corresponding value $h$, the stability spectrum (the set of roots of the characteristic equation (2.2)) contains the pair of purely imaginary roots $\pm i \sigma$, where $\sigma>0$.

The corresponding pairs $(h, \sigma)$ are defined as solutions of the system

$$
(k-1) \cos \sigma h=0, \quad \sigma=(1-k) \sin \sigma h .
$$

In this case, $k-1 \neq 0(k \in(0,1))$. Therefore, we finally obtain a system for determining $H$ and $\sigma$ already of the following form

$$
\cos \sigma h=0, \quad \sigma h=(1-k) h \sin \sigma h
$$

or after relabeling $\omega=\sigma h$, the system (2.3) can be written in the following form

$$
\cos \omega=0, \quad(1-k) h \sin \omega=\omega .
$$

Observe that system (2.4) has the following set of solutions

$$
\omega_{m}=\frac{\pi}{2}+\pi m, \quad h_{m}=\frac{\omega_{m}}{(1-k) \sin \omega_{m}}, \quad m=0, \pm 1, \pm 2, \ldots
$$

The smallest $H=\min \left\{h_{m}\right\}>0$ is possible if

$$
\omega=\frac{\pi}{2}, \quad H=\frac{\pi}{2(1-k)}, \quad \sigma=1-k .
$$


Applying this terminology for $H=\frac{\pi}{2(1-k)}$, the stability spectrum contains a pair of pure imaginary roots $\lambda_{1,2}= \pm i \sigma$ and for the remaining $\lambda_{k}$ the inequality $\operatorname{Re} \lambda_{k}<0$ is valid.

Now let $h=H(1+\gamma \varepsilon)$. Then from the characteristic equation (2.2) we obtain $\lambda_{0}^{\prime}=\left.\frac{d \lambda(\varepsilon)}{d \varepsilon}\right|_{\varepsilon=0}=\tau_{0}^{\prime}+i \sigma_{0}^{\prime}$, and the equality

$$
\lambda_{0}^{\prime}=-i \sigma H \lambda_{0}^{\prime}+H \sigma^{2} \gamma,
$$

which allows us to determine $\lambda_{0}^{\prime}$. In our case we acquire the following equality:

$$
\tau_{0}^{\prime}=\frac{2 \pi(1-k)}{4+\pi^{2}} \gamma
$$

Note that if we choose the constant $\gamma=1$, then the inequality $\tau_{0}^{\prime}>0$ will hold true. In this case, as $h$ increases, the roots $\pm i \sigma$ go to the right half-plane of the complex plane. Consequently, the zero solution of the auxiliary equation (1.7) loses its stability; in particular, the existence of a stable cycle is possible. The last question will be addressed in the next section.

Analogous constructions must also be implemented for equations (1.8) and (1.9). Thus, for equation (1.8) we acquire the following version of the linearized equation:

$$
\dot{u}=-v+k u \text {. }
$$

The stability question of the solutions of Equation (2.5) reduces to an analysis of the following characteristic equation:

$$
\lambda=-\exp (-\lambda h)+k .
$$

When $h=0$, we have the root $\lambda=k-1<0$. To find the positive $\min h=H$, for which roots with $\operatorname{Re} \lambda=0$ emerge in equation (2.6). The case $\lambda=0$ is impossible for any $h$. Therefore, the critical case in the stability problem is possible if the corresponding value $h$, the stability spectrum (the set of roots of the characteristic equation (2.6)) has a pair of pure imaginary roots $\pm i \sigma$, where $\sigma>0$. The corresponding pairs $(h, \sigma)$ are defined as solutions of the following system

$$
0=k-\cos \sigma h, \quad \sigma=\sin \sigma h .
$$

Now let $\sigma h=\omega(h \neq 0)$. Then $\omega_{m}=\arccos k+2 \pi m, h_{m}=\frac{\omega_{m}}{\sin \omega_{m}}$, or $\omega_{p}=$ $-\arccos k+2 \pi p, h_{p}=\frac{\omega_{p}}{\sin \omega_{p}}$, where $m, p \in Z$ is the set of integers. An elementary analysis of the previous two equalities confirms that the smallest $H=h>0$ is defined by the following equality:

$$
H=\frac{\arccos k}{\sqrt{1-k^{2}}}=\frac{a}{\sqrt{1-k^{2}}}, \quad a=\arccos k .
$$

Moreover, the corresponding $\sigma=\sqrt{1-k^{2}}>0$. Naturally, for $h=H$ there is a conjugate root $-i \sigma$.

We will show that with increasing $h$, i.e. for $h>H$ the roots $\pm i \sigma$ of the characteristic equation go to the right half-plane. Then to this end, we set 
$h=H(1+\varepsilon)$, where $\varepsilon$ is a sufficiently small parameter. For such $h=h(\varepsilon)$ we acquire the following characteristic equation

$$
\lambda(\varepsilon)=k-\exp (-\lambda(\varepsilon)(1+\varepsilon) H) .
$$

From where we then determine

$$
\lambda_{0}^{\prime}=\left.\frac{d \lambda(\varepsilon)}{d \varepsilon}\right|_{\varepsilon=0}=\frac{i \sigma H(k-i \sigma)}{(1-H k)+i H \sigma}
$$

and, consequently, we obtain that $\operatorname{Re} \lambda_{0}^{\prime}=\frac{H \sigma^{2}}{(1-H k)^{2}+H^{2} \sigma^{2}}>0$, with increasing $h(h>H)$, the roots $\pm i \sigma$ go to the right half-plane $\operatorname{Re} \lambda>0$. We emphasize that for small $\varepsilon$ the remaining roots of the characteristic equation lie in the half-plane $\operatorname{Re} \lambda \leq-\gamma_{0}<0$, where $\gamma_{0}$ is independent of $\varepsilon$. This result follows from the results presented in the monographs $[1,7]$.

During the analysis of the linearized equation (1.9), we acquire the following characteristic equation

$$
\lambda=-1+k \exp (-\lambda h), \quad k \in(0,1) .
$$

Note when $h=0$, we get $\lambda=-1+k<0$. We show that this equation cannot have roots with $\operatorname{Re} \lambda=0$. In fact, the root $\lambda=0$ is impossible, as we obtain the equality $k=1$. The previous equation cannot have roots in the form $\pm i \sigma$. Indeed, this assumption leads us to the following system

$$
\cos \sigma h=1 / k, \quad k \sin \sigma h=-\sigma .
$$

There is no solution as $1 / k>1$ by assumption. Hence it follows that the characteristic equation does not have roots with $R e \lambda>0$. Therefore as a result, it is shown that further study of equation (1.9) is unsubstantiated. Such an option of introducing delay does not bring new effects in comparison with the analysis of the ordinary differential equation (1.2).

In the next two sections, equations (1.7) and (1.8) will be analyzed in a nonlinear formulation on the basis of the application of the Andronov-Hopf theorem for equations with deviating argument. The proof of the AndronovHopf theorem itself for equations with a deviating argument can be found in $[7,12,17]$. Our goals of this paper are to present a modern version of the presentation of this question using the modified Krylov-Bogolyubov algorithm and the theory of normal forms of Poincare-Dulac. This method of analyzing macroeconomic models was applied in $[15,16,18,19]$.

\section{Periodic solutions}

Our intents of this section are to study the existence and stability of cycles of equation (1.7). The existence and stability of cycles of equation (1.8) will be in the next section. In equation (1.6) we set:

$$
t=\frac{h(\varepsilon)}{H} \Theta, h(\varepsilon)=H(1+\gamma \varepsilon), \gamma \in R, \varepsilon \in\left(0, \varepsilon_{0}\right), 0<\varepsilon_{0}<<1, H=\frac{\pi}{2(1-k)} .
$$


Notice that $\Theta$ is a new normalized time. As a result of such a time change, equation (1.7) is rewritten in the form

$$
u^{\prime}=(1+\gamma \varepsilon)\left[-v+k v+\frac{k(k-1)}{2} v^{2}+\frac{k(k-1)(k-2)}{6} v^{3}+o\left(v^{3}\right)\right],
$$

where $u=u(\Theta), v=u(\Theta-H)$, and the prime in the equation (3.1) denotes the derivative with respect to the variable $\Theta$.

In the neighborhood of the zero equilibrium state equation (3.1) has a twodimensional smooth invariant manifold $M_{2}(\varepsilon)[6,8,14]$. In this case, all solutions of equation (3.1) approach it with the velocity of the exponent with time if their initial conditions are small in the sense of the norm of the phase space of solutions. Recall that in our case this is $C[-H, 0](C[-H, 0]$; the space of continuous functions $g(\Theta)$ on $[-H, 0]$ with the norm $\left.\|g\|=\max _{\Theta \in[-H, 0]}|g(\Theta)|\right)$, and the dynamics of the solutions of equation (3.1) is restored after analyzing the system of two ordinary differential equations - normal form (NF). In the assumed case, NF can be written in complex form $[15,16,18,19]$

$$
z^{\prime}=(\alpha+i \beta) z+(d+i c) z|z|^{2}+O(\varepsilon)
$$

where $\alpha, \beta, d, c \in R$. These coefficients can be written out in an explicit form, which will be done below after the implementation of the algorithm for constructing the defining equation, which is commonly called NF. These coefficients depend on the parameters of equation (3.1). In our case, these are $k$ and $H$. For this purpose, recently it has been customary to use the adaptation of the Krylov-Bogolyubov algorithm to infinite-dimensional dynamical systems $[15,16,18,19]$. In the $\mathrm{NF}(3.2), z=z(s)$ is a complex function, and $s=\varepsilon \Theta, \varepsilon \in\left(0, \varepsilon_{0}\right)$. If we assume that the first Lyapunov value $d(d \neq 0)$ is non-zero a priori, then the solution of Equations (3.1) with initial conditions in a small neighborhood of the zero solution can be expediently sought in the following form $[15,16,18,19]$

$$
u(\Theta, \varepsilon)=\varepsilon^{1 / 2} u_{1}(\Theta, z)+\varepsilon u_{2}(\Theta, z)+\varepsilon^{3 / 2} u_{3}(\Theta, z)+O\left(\varepsilon^{2}\right),
$$

where $u_{1}(\Theta, z)=z(s) \exp (i \sigma \Theta)+\bar{z}(s) \exp (-i \sigma \Theta), z(s)$ - one of the solutions of the NF (see Section 3). The sufficiently smooth functions $u_{2}(\Theta, z), u_{3}(\Theta, z)$ with respect to the variable $\Theta$ have period $2 \pi / \sigma$ and in addition we obtain

$$
M_{ \pm}\left(u_{m}\right)=\frac{\sigma}{2 \pi} \int_{0}^{2 \pi / \sigma} u_{m}(\Theta, z) \exp ( \pm i \sigma \Theta) d \Theta=0
$$

for $m=2,3$ and any $z(s)$ is assumed. Note that $v(\Theta, \varepsilon)=u(\Theta-H, \varepsilon)$, and also that $O\left(\varepsilon^{2}\right)$ denote the function $\psi(z, \bar{z}, \varepsilon)$ for which the estimate

$$
|\psi(z, \bar{z}, \varepsilon)| \leq M \varepsilon^{2}[|z|+|\bar{z}|], M>0
$$

is valid. We substitute the sum (3.3) into Equation (3.1) and equate the coefficients for the powers $\varepsilon, \varepsilon^{3 / 2}$. Hence we obtain two linear nonhomogeneous 
delayed differential equations. Thus, to determine $u_{2}(\Theta, z)$, we obtain the following equation

$$
\frac{\partial u_{2}}{\partial \Theta}+(1-k) v_{2}=\Phi_{2}(\Theta, z)
$$

where $\Phi_{2}(\Theta, z)=\frac{k(k-1)}{2} v_{1}^{2}, v_{2}=u_{2}(\Theta-H, z), v_{1}(\Theta)=u_{1}(\Theta-H)$. When deriving the equation for $u_{3}(\Theta, z)$, one can note that $\frac{d}{d \Theta} \psi(\Theta, s)=\frac{\partial \psi}{\partial \Theta}+\frac{\partial \psi}{\partial s} \varepsilon$. Therefore, we obtain the following equation

$$
\begin{aligned}
& \frac{\partial u_{3}}{\partial \Theta}+v_{3}-k v_{3}=\Phi_{3}(\Theta, z) \\
& \Phi_{3}(\Theta, z)=k(k-1) v_{1} v_{2}+\frac{k(k-1)(k-2)}{6} v_{1}^{3}+\gamma(k-1) v_{1} \\
& \quad-z^{\prime} i \frac{\pi}{2} \exp (i \sigma \Theta)+\bar{z}^{\prime} i \frac{\pi}{2} \exp (-i \sigma \Theta)-z^{\prime} \exp (i \sigma \Theta)-\bar{z}^{\prime} \exp (-i \sigma \Theta), \\
& z=z(s), \quad \sigma=1-k, \quad z^{\prime}=\frac{d z}{d s}, \quad v_{1}=-i z \exp (i \sigma \Theta)+i \bar{z} \exp (-i \sigma \Theta) .
\end{aligned}
$$

In the previous formulas we use the equality $H=\frac{\pi}{2(1-k)}, \exp \left(i \frac{\pi}{2}\right)=i$.

Comment.Linear differential equation with a deviating argument

$$
\frac{d u}{d \Theta}+(1-k) u(t-H)=\Phi(\Theta)
$$

where $H=\frac{\pi}{2(1-k)}, \Phi(\Theta)-$ a periodic function of the variable $\Theta$ with period $2 \pi / \sigma$ has periodic solutions with the same period if

$$
M_{ \pm}(\Phi(\Theta))=\frac{\sigma}{2 \pi} \int_{0}^{2 \pi / \sigma} \Phi(\Theta) \exp ( \pm i \sigma \Theta) d \Theta=0 .
$$

The equalities of $M_{ \pm}(u)=0$ single one such solution.

Therefore, in the case of equation (3.4), when

$$
\Phi_{2}(\Theta, z)=\frac{k(1-k)}{2}\left[z^{2} \exp (2 i \sigma \Theta)-2|z|^{2}+\bar{z}^{2} \exp (-2 i \sigma \Theta)\right],
$$

the solution of this equation should be selected in the following form

$$
u_{2}(\Theta, z)=\eta_{2} z^{2} \exp (2 i \sigma \Theta)+\eta_{0}|z|^{2}+\bar{\eta}_{2} \bar{z}^{2} \exp (-2 i \sigma \Theta),
$$

where $\eta_{2} \in C, \eta_{0} \in R$. After substituting $u_{2}(\Theta, z)$ in the selected form in the corresponding equation we determine that

$$
\eta_{0}=-k, \eta_{2}=-\frac{k}{10}(1+2 i)
$$

We now analyze the nonhomogeneous differential equation (3.5). From the solvability conditions for this equation in the class $2 \pi / \sigma$ of periodic functions, we obtain the following equality

$$
\frac{k(1-k)}{5}[k-(3 k+5) i] z|z|^{2}-\gamma i(k-1) z-\left(1+i \frac{\pi}{2}\right) z^{\prime}=0 .
$$


This equation should be transformed to a standard notation for differential equations by selecting $z^{\prime}$. Hence we obtain the following equation:

$$
z^{\prime}=(\alpha+i \beta) z+(d+i c) z|z|^{2} .
$$

In our case we get

$$
\begin{aligned}
& \alpha=2 \pi \gamma \frac{1-k}{4+\pi^{2}}, \quad \beta=4 \gamma \frac{1-k}{4+\pi^{2}}, \\
& d=-\frac{4 k(1-k)}{5\left(4+\pi^{2}\right)}\left[\left(\frac{3 \pi}{2}-1\right) k+\frac{5 \pi}{2}\right], \quad c=-\frac{4 k(1-k)}{5\left(4+\pi^{2}\right)}\left[5+\left(3+\frac{\pi}{2}\right) k\right] .
\end{aligned}
$$

We emphasize that equation (3.6) is a "shorter" version of the NF. Note that for all $k$ under the assumption of the first Lyapunov value $d<0$.

Lemma 1. Equation (3.6) has a periodic solution $z(s)=\rho \exp (i \nu s)$, where $\rho=\sqrt{\gamma \frac{5 \pi}{2 k}\left[\left(\frac{3 \pi}{2}-1\right) k+\frac{5 \pi}{2}\right]^{-1}}, \nu=\beta+c \rho^{2}$, if $\gamma>0$. Moreover, this periodic solution is stable while the zero solution is unstable. For $\gamma<0$ this equation has no non-trivial periodic solutions and the zero solution of the NFis asymptotically stable.

The proof of the lemma is trivial. In fact, similar statements can be found in many textbooks and monographs (see, for example, $[6,8]$ ).

Now by substituting $z(s)=\rho \exp (i \nu s)$ into Equation (3.6) leads to a system of algebraic equations for determining $\rho$ and $\nu$

$$
\alpha \rho+d \rho^{3}=0, \quad \nu=\beta+c \rho^{2} .
$$

The first equation of the given system has a non-trivial solution $\rho>0$, if $\alpha$ and $d$ of different signs and only the zero solution if the signs $\alpha, d$ coincide. The stability analysis of a non-trivial periodic solution is described by the standard scheme. Now we set $z=\rho \exp (i \nu s)(1+w)$. The linearized version for the vector-valued function $\eta=\left(w_{1}, w_{2}\right)\left(w=w_{1}+i w_{2}\right)$ has the following form $\eta^{\prime}=2 B \eta$, with the following matrix

$$
B=\left(\begin{array}{ll}
d \rho^{2} & 0 \\
c \rho^{2} & 0
\end{array}\right) .
$$

One eigenvalue of the matrix is $\lambda_{1}=d \rho^{2}<0$, if $d<0(\alpha>0)$ and this is an eigenvalue of $\lambda_{1}>0$, if $d>0(\alpha<0)$, and the second eigenvalue of the matrix is $\lambda_{2}=0$. The Andronov-Witt theorem implies the validity of the lemma's assertion. We emphasize that a simpler version of the proof of the lemma's assertion is compared with the earlier papers. The results from $[10,11,12,14,17]$ simply the validity of the assertion.

Theorem 1. There exists $\varepsilon_{0}>0$, such that for all $\varepsilon \in\left(0, \varepsilon_{0}\right)$ equation (3.1) for $\gamma>0$ has a stable (orbitally asymptotically stable) limit cycle corresponding to the NF cycle with the following asymptotic formula

$$
\begin{aligned}
& u_{*}(\Theta, \varepsilon)=\varepsilon^{1 / 2} \rho[\exp (i(\sigma+\varepsilon \nu) \Theta+i \varphi)+\exp (-i(\sigma+\varepsilon \nu) \Theta-i \varphi)] \\
& \quad+\varepsilon \rho^{2}\left[\eta_{2} \exp (2 i(\sigma+\varepsilon \nu) \Theta+2 i \varphi)+\eta_{0}+\bar{\eta}_{2} \exp (-2 i(\sigma+\varepsilon \nu) \Theta-2 i \varphi)\right]+o(\varepsilon),
\end{aligned}
$$

where $\varphi \in R$ and the constants $\rho, \sigma, \nu, \eta_{0}, \eta_{2}$ were indicated earlier. 
These substitutions allow us to transfer the results of the Theorem 1 to equation (1.5). Now let $h=H(1+\varepsilon)$. Then the periodic solution $u_{*}(\Theta, \varepsilon)$ corresponds to the periodic solution of equation (1.5)

$$
x_{*}(t, \varepsilon)=1+u_{*}\left(\frac{t}{1+\varepsilon}, \varepsilon\right)(\gamma=1) .
$$

Naturally, the solution $x_{*}(t, \varepsilon)$ is stable in the sense of A.M. Lyapunov.

Also notice that the periodic solutions depend on the selection of the parameter $k$. For instance when $k=1-\delta$ and $\delta$ is sufficiently small, we obtain periodic solutions with long periods that can be interpreted as "long waves", N.D. Kondratiev (see also [13,20]).

\section{Nonlinear analysis of an alternative version of the modified version of the Solow equation}

In this section we will analyze Equation (1.8). Similar to the previous section, we set

$$
t=\frac{h(\varepsilon)}{H} \Theta, h(\varepsilon)=H(1+\gamma \varepsilon), \gamma \in R, \varepsilon \in\left(0, \varepsilon_{0}\right), 0<\varepsilon_{0}<<1, H=\frac{\pi}{2(1-k)} .
$$

$\Theta$ is a new normalized time. As a result of this time change, Equation (1.8) is rewritten as follows

$$
u^{\prime}=(1+\gamma \varepsilon)\left[-v+k u+\frac{k(k-1)}{2} u^{2}+\frac{k(k-1)(k-2)}{6} u^{3}+o\left(u^{3}\right)\right],
$$

where $u=u(\Theta), v=u(\Theta-H)$, and the prime in the Equation (4.1) denotes the derivative with respect to the variable $\Theta$ in the new normalized time.

In the neighborhood of the zero equilibrium state, equation (4.1) has a two-dimensional smooth invariant manifold $M_{2}(\varepsilon)$. In this case, if their initial conditions are small in the sense of the norm of the phase space of solutions, then all the solutions of equation (4.1) approach it with the velocity of the exponent with time. Recall that in our case the phase space is $C[-H, 0](C[-H, 0]$ is the space of continuous functions on $[-H, 0]$, and the functions $g(\Theta)$ with the norm $\left.\|g\|=\max _{\Theta \in[-H, 0]}|g(\Theta)|\right)$, and the dynamics of the solutions of equation (4.1). It can be restored after the analysis of the system of two ordinary differential equations (NF). In this case, the NF can be written in complex form and has the same structure as in the previous section

$$
z^{\prime}=(\alpha+i \beta) z+(d+i c) z|z|^{2}+O(\varepsilon),
$$

where $\alpha, \beta, d, c \in R$. These coefficients can be written out in an explicit form, which will be performed below after the implementation of the algorithm for constructing the determining equation (NF). If we assume that a priori $d \neq 0$, then the solution of equation (4.1) with the set of initial conditions from a small neighborhood of the zero solution it is possible and expedient to look for in the following form (see Section 3)

$$
u(\Theta, \varepsilon)=\varepsilon^{1 / 2} u_{1}(\Theta, z)+\varepsilon u_{2}(\Theta, z)+\varepsilon^{3 / 2} u_{3}(\Theta, z)+O\left(\varepsilon^{2}\right),
$$


where $u_{1}(\Theta, z)=z(s) \exp (i \sigma t)+\bar{z}(s) \exp (-i \sigma t), z(s)$ - one of the solutions of the NF. The sufficiently smooth functions $u_{2}(\Theta, z), u_{3}(\Theta, z)$ with respect to the variable $\Theta$ have period $2 \pi / \sigma$, where $\sigma$ is selected after the analysis of the corresponding linearized equation in the second section.

We substitute the sum (4.2) into equation(4.1) and equate the coefficients for the powers $\varepsilon, \varepsilon^{3 / 2}$. As a result, we obtain two linear nonhomogeneous delayed differential equations. Thus, to determine $u_{2}(\Theta, z)$, we get the following equation

$$
\frac{\partial u_{2}}{\partial \Theta}+v_{2}-k u_{2}=\Phi_{2}(\Theta, z)
$$

where $\Phi_{2}(\Theta, z)=\frac{k(k-1)}{2} u_{1}^{2}, v_{2}=u_{2}(\Theta-H, z)$, and

$$
\frac{\partial u_{3}}{\partial \Theta}+v_{3}-k u_{3}=\Phi_{3}(\Theta, z)
$$

where

$$
\begin{gathered}
\Phi_{3}(\Theta, z)=k(k-1) u_{1} u_{2}+\frac{k(k-1)(k-2)}{6} u_{1}^{3}+\gamma\left[-v_{1}+k u_{1}\right] \\
+z^{\prime} H \exp (-i \sigma H) \exp (i \sigma \Theta)+\bar{z}^{\prime} H \exp (i \sigma H) \exp (-i \sigma \Theta) \\
-z^{\prime} \exp (i \sigma \Theta)-\bar{z}^{\prime} \exp (-i \sigma \Theta), \quad z=z(s), \quad z^{\prime}=\frac{d z}{d s}
\end{gathered}
$$

In this case we obtain the following solution of equation (4.3)

$$
u_{2}(\Theta, z)=\eta_{2} z^{2} \exp (2 i \sigma \Theta)+\eta_{0}|z|^{2}+\bar{\eta}_{2} \bar{z}^{2} \exp (-2 i \sigma \Theta),
$$

where

$$
\eta_{0}=-k, \quad \eta_{2}=\eta_{21}+i \eta_{22}, \quad \eta_{21}=\frac{k(2 k+1)}{2(5+4 k)}, \quad \eta_{22}=\frac{k \sqrt{1-k^{2}}}{5+4 k} .
$$

From the analysis of equation (4.4) we acquire

$$
\begin{aligned}
& \alpha=\gamma \frac{\left(1-k^{2}\right)^{3 / 2} a}{Q}, \quad \beta=\frac{1-k^{2}}{Q} \gamma\left(\sqrt{1-k^{2}}-a k\right), \quad d=\frac{k\left(1-k^{2}\right)^{3 / 2}}{(5+4 k) Q} \\
& \times\left[(k+5) \sqrt{1-k^{2}}-6 a k\right], c=-\frac{k\left(1-k^{2}\right)^{2}}{(5+4 k) Q(1+k)}\left[k \sqrt{1-k^{2}}+a(6 k+5)\right], \\
& Q=\left(\sqrt{1-k^{2}}-a k\right)^{2}+a^{2}\left(1-k^{2}\right)>0 .
\end{aligned}
$$

In the case when $a>0$, we immediately notice that the $\operatorname{sign} \alpha$ coincides with the sign $\gamma$, and the sign of the Lyapunov value $d$ is determined by the sign of the factor $\varphi(k)=(k+5) \sqrt{1-k^{2}}-6 a k$, as the remaining factors in the formula for $d$ are certainly positive. It is easy to see that $\varphi(k)>0$ for all $k \in(0,1)$. Obviously, we obtain $\varphi(k)=\varphi(a)=(5+\cos a) \sin a-6 a \cos a$, where $a=\arccos k(a \in(0, \pi / 2))$. The sign, $\varphi(a)$ coincides with the sign of the function

$$
\psi(a)=\frac{\varphi(a)}{\cos a}=5 \tan a+\sin a-6 a,
$$


where $\psi(0)=0$, a $\psi^{\prime}(a)=\frac{5}{\cos ^{2} a}+\cos a-6>0$ for all $a \in(0, \pi / 2)$, and

$$
\psi^{\prime}(a) \geq \frac{1}{\cos ^{2} a}\left(5+\cos ^{4} a-6 \cos ^{2} a\right)=\frac{1}{\cos ^{2} a}\left(5 \sin ^{2} a-\sin ^{2} a \cos ^{2} a\right)>0,
$$

where $a \in(0, \pi / 2)$. Therefore, in the case of equation (1.8) when the first Lyapunov value $d>0$, then the NF has a periodic solution if $\alpha<0(\gamma=-1)$

$$
z=z(s)=\rho \exp (i \omega s), \quad \rho=\sqrt{-\alpha / d},
$$

and $\alpha, d, \beta$ are indicated above and hence this solution is unstable. Consequently, the following assertion is true.

Theorem 2. Suppose that $k \in(0,1-\delta), \delta>0$. Then there exists $\varepsilon_{0}=\varepsilon_{0}(\delta)>$ 0 , that for all $\varepsilon \in\left(0, \varepsilon_{0}\right)$ Equation (4.1) has an unstable limit cycle for $h=$ $(1-\varepsilon) H$. For the corresponding periodic solutions we acquire the following asymptotic formula

$$
\begin{aligned}
& u_{*}(\Theta, \varepsilon)=\varepsilon^{1 / 2} \rho\left[\exp \left(i(\sigma+\varepsilon \omega) \Theta+i \varphi_{0}\right)+\exp \left(-i(\sigma+\varepsilon \omega) \Theta-i \varphi_{0}\right)\right] \\
& +\varepsilon \rho^{2}\left[\eta \exp \left(2 i(\sigma+\varepsilon \omega) \Theta+2 i \varphi_{0}\right)-k+\bar{\eta} \exp \left(-2 i(\sigma+\varepsilon \omega) \Theta-2 i \varphi_{0}\right)\right]+o(\varepsilon) .
\end{aligned}
$$

$\varphi_{0} \in R$, the constants $\rho, \eta$ were specified earlier.

It is pertinent to note that the unstable cycles exist when $k \in(0,1-\delta)$. For $k \rightarrow 1-0$, the amplitude $\rho=\rho(k) \rightarrow \infty$. Consequently, the case when $k \rightarrow 1-0$ requires additional investigation. Certainly, for such $k$ and $\sigma=\sigma(k)$ the limit equality $\lim _{k \rightarrow 1-0} \sigma(k)=0$ also holds. Finally, equation (1.3) exhibits the following periodic solutions

$$
x_{*}(t, \varepsilon)=1+u_{*}\left(\frac{t}{1-\varepsilon}, \varepsilon\right) .
$$

In this case we acquired a family of periodic solutions, since $u_{*}(\Theta, \varepsilon)$ depends on an arbitrary constant $\varphi_{0} \in R$. All these solutions are naturally unstable.

\section{Conclusions}

In this paper we have shown that the delay effects can significantly change the dynamics of solutions in the classical Solow model. At least in two of the presented forms of the correspondingly modified Solow Equation may appear to fluctuate the variable capital-labor, which is typical for pricing within a market economy. Especially the introduction of a delay was very informative in both terms on the right-hand side of the Solow equation. If we consider the delay factor for depreciation of funds and investments at the same time, a stable cycle will emerge in the equation that describes the cyclical nature of the price change and on a qualitative level explains the cyclic character inherent in the economic processes.

In the second form, when the delay is introduced only in one of the terms responsible for the depreciation of funds, the cycle is unstable but the price 
fluctuations are possible. Recall that in the classic version of the Solow model the price fluctuations are impossible. Solutions of the corresponding equation $p(\tau) \rightarrow p_{0}>0$ are monotonic functions. For $p(0)<p_{0}, p(\tau)$ increases and decreases as $p(0)>p_{0}$, where $p_{0}$ is the equilibrium price.

A similar effect occurs if a delay is introduced into another classical model of macroeconomics - the "supply-demand" model (market model). In this classical version, there are no oscillatory solutions but the assumption of the delay showed that under certain variations periodic cycles may exist. The selection of the parameters of the problem has a modified version of the equation that already has a periodic cycle (see $[15,18,19])$.

In macroeconomics, a similar situation is reproduced for the one that occurred in mathematical ecology. If we consider the well-known Verhulst equation

$$
\dot{N}=\alpha N(1-N)
$$

then of course it does not exhibit periodic solutions. Notice that $\alpha>0, N=$ $N(t)$ resembles the number of species. This is a significant contribution by Hutchinson [9], who proposed to study the following equation

$$
\dot{N}=\alpha N(1-N(t-h)), h>0,
$$

which is already capable of describing fluctuations in the population size in a single-species bio-sinopsis.

\section{Acknowledgements}

The study was conducted with the financial support of the Russian Foundation for Basic Research in the framework of the scientific project No 18-01-00672.

\section{References}

[1] R. Bellman and K.L. Cooke. Differential-Difference Equations. Academic press, London, 1963.

[2] C. Bianca and L. Guerrini. Existence of limit cycles in the Solow model with delayed-logistic population growth. The Scientific World Journal, 2014:1-8, 2014. https://doi.org/10.1155/2014/207806.

[3] M. Ferrara, L. Guerrini and R. Mavilla. Modified neoclassical growth models with delay: a critical survey and perspectives. Applied Mathematical Sciences, 7(86):4249-4257, 2013. https://doi.org/10.12988/ams.2013.36318.

[4] M. Ferrara, L. Guerrini and M. Sodini. Nonlinear dynamics in a Solow model with delay and non-convex technology. Applied Mathematics and Computation, 228:1-12, 2014. https://doi.org/10.1016/j.amc.2013.11.082.

[5] L. Gori, L. Guerrini and M. Sodini. A model of economic growth with physical and human capital: The role of time delays. Chaos, 16(4):093118, 2016. https://doi.org/10.1063/1.4963372.

[6] J. Guckenheimer and P.J. Holmes. Nonlinear Oscillations, Dynamical Systems, and Bifurcations of Vector Fields. Springer-Verlag, Berlin-New-York, 1983. 
[7] J. Hale. Theory of functional differential equations. Springer-Verlag, Berlin-NewYork, 1977.

[8] M. Haragus and G. Iooss. Local Bifurcations, Center Manifolds and Normal Forms in Infinite Dimensional Dynamical Systems. EDP Sciences, Berlin, 2011.

[9] G.E. Hutchinson. Circular causal systems in ecology. Annals of the NewYork Academy of Sciences, 50(4):221-246, 1948. https://doi.org/10.1111/j.17496632.1948.tb39854.x.

[10] A.Yu. Kolesov, A.N. Kulikov and N.Kh. Rozov. Invariant tori of a class of point mappings: the annulus principle. Differ. Equ., 39(5):614-631, 2003. https://doi.org/10.1023/A:1026133701786.

[11] A.Yu. Kolesov, A.N. Kulikov and N.Kh. Rozov. Invariant tori of a class of point transformations: preservation of an invariant torus under perturbations. Differ. Equ., 39(6):775-790, 2003. https://doi.org/10.1023/B:DIEQ.0000008405.33370.ff.

[12] Yu.S. Kolesov. Harmonic self-oscillations of n-order differential equations with after effect. Vestnik Yaroslavl. univ., 7:3-88, 1974.

[13] N.D. Kondratiev. Special opinion. Book 2. Nauka, Moscow, 1993.

[14] A.N. Kulikov. On smooth invariant manifolds of nonlinear operator in the Banach space. Studies of stability and the theory of oscillation, pp. 67-85, 1976.

[15] A.N. Kulikov and D.A. Kulikov. The effect of delay and the economic cycles. Taurida Journal of Computer Sciences Theory and Mathematics, 2(27):87-100, 2015.

[16] A.N. Kulikov and D.A. Kulikov. The mathematical model of the market and the effect of delay. Mathematica $v$ Yaroslavskom univ.Sbornik Obsor. Statey $k$ 40-let. Matem. Faculty, pp. 132-150, 2016.

[17] A.N. Kulikov and D. Shvitra. Bifurcations of small periodic solutions of differential equations with delay of neutral type. Differential equations and applications, 16:41-59, 1976.

[18] D.A. Kulikov. About a mathematical model of market. IOP Conf. Series: Journal of Physics: Conference Series, 788:6p., 2017. https://doi.org/10.1088/17426596/788/1/012024.

[19] D.A. Kulikov. Stability and local bifurcations of the Solow model with delay. Zhurnal SVMO, 20(2):225-234, 2018. https://doi.org/10.15507/2079$6900.20 .201802 .225-234$.

[20] V.V. Lebedev and K.V. Lebedev. Mathematical modelling of nonstationary processes. eTest, Moscow, 2011.

[21] R.M. Solow. A contribution to the theory of economic growth. The Quarterly Journal of Economics, 70(1):65-94, 1956. https://doi.org/10.2307/1884513.

[22] T.W. Swan. Economic growth and capital accumulation. Economic Record, 32:334-361, 1956. https://doi.org/10.1111/j.1475-4932.1956.tb00434.x.

[23] M. Szydlowski and A. Krawiec. A mote on Kaleckian lags in the Solow model. Review of Political Economy, 16(4):501-506, 2004. https://doi.org/10.1080/0953825042000256711.

[24] P.J. Zak. Kaleskian lags in general equilibrium. Review of Political Economy, 11(3):321-330, 1999. https://doi.org/10.1080/095382599107048.

[25] W.B. Zhang. Synergetic Economics: Time and Change in Nonlinear Economics. Springer-Verlag, Berlin-New-York, 1983. 